\title{
Introdução à mecânica dos quanta Parte IV ${ }^{1}$
}

Theodoro Ramos

\section{Escola Politécnica de São Paulo}

\begin{abstract}
Neste quarto artigo o autor introduz a mecânica matricial de Heisenberg e mostra sua equivalência com a mecânica ondulatória de Schrödinger.

Palavras-chave: mecânica matricial.

In the fourth paper of the series, the author introduces the matrix mechanics due to Heisenberg and shows its equivalence to the Schrödinger's undulatory mechanics.
\end{abstract}

Keywords: matrix mechanics.

\section{Noções sobre o cálculo e a mecânica das matrizes}

Heisenberg, ao elaborar, em 1925, a nova Mecânica dos "Quanta", procurou fixar a sua atenção unicamente sobre as grandezas que possuem significação experimental precisa, e tais como: do átomo;

1) as freqüências e as intensidades das raias espectrais

2) os níveis energéticos do átomo que nos são revelados, quer pelas experiências sobre o choque eletrônico (experiências de Frank e Hertz, e de outros), quer pela espectroscopia que nos fornece, para o cálculo da freqüência da radiação emitida pelo átomo, a fórmula fundamental $v_{n m}=T_{n}-T_{m}$; os termos espectroscópicos $T_{n}$ e $T_{m}$, sendo associados aos níveis energéticos $E_{n}$ e $E_{m}$.

Na elaboração da nova Mecânica Atômica, valeu-se Heisenberg largamente do principio de correspondência de Bohr já examinado na última conferência.

Consideremos primeiramente o caso de um sistema atômico dependendo de um único parâmetro (real) $x$.

Vimos em uma conferência anterior que na teoria eletromagnética clássica quando se estuda a radiação emitida por um átomo procura-se desenvolver $x(t)$ em série de Fourier

$$
x(t)=\sum_{\tau} a_{\tau} \exp (2 \pi i \tau v t)
$$

$\tau$ tomando os valores inteiros de $-\infty$ a $+\infty$. Os coeficientes $\mathrm{a}_{\tau}$ dependem da variável de "ação" $J$, e como $x$ é real devemos ter $a_{-\tau}=a_{\tau}^{*}$ ou $a_{\tau}=a_{-\tau}^{*} \quad$ (o asterisco significando o imaginário conjugado). $v$ é a freqüência fundamental da vibração do elétron: $\mathrm{a}_{\tau}$ é a amplitude complexa da componente harmônica de ordem $\tau$. A intensidade da harmônica de ordem $\tau$ é proporcional a $\left|a_{\tau}\right|^{2}=a_{\tau} a_{-\tau}$.

A teoria clássica faz corresponder à variável dinâmica $x(t)$ uma sucessão de quantidades $\mathrm{a}_{\tau} \exp (2 \pi \mathrm{i} \tau \nu t)$ cujo conhe- cimento acarreta o de $x(t)$ por intermédio da série de Fourier.

Na nova Mecânica Atômica, Heisenberg, guiado pelo principio de correspondência de Bohr, caracterizou a variável $x$ pelo conjunto de quantidades $x_{n m}=a_{n m} \exp \left(2 \pi \mathrm{i} v_{n m} t\right)$, ( $m=n-\tau)$, dispostas no quadro

$$
\left(x_{n m}\right)=\left(\begin{array}{cccc}
x_{00} & x_{01} & x_{02} & \ldots \\
x_{10} & x_{11} & x_{12} & \ldots \\
x_{20} & x_{21} & x_{22} & \ldots \\
\vdots & \vdots & \vdots & \ddots
\end{array}\right)
$$

em que $v_{n m}$ é a frequiência da radiação emitida pelo átomo quando passa do estado energético $n$ ao estado $m=n-\tau$; $\left|a_{n m}\right|^{2}$ é proporcional à intensidade da radiação emitida, e, na linguagem estatística de Born, proporcional à probabilidade de passagem do átomo do estado $n$ ao estado $m$.

Escreve-se $x=\left(x_{n m}\right)$. Como $x$ é real, supõe-se que $a_{n m} \mathrm{e}$ $a_{m n}$ são imaginários conjugados, e que, portanto

$$
x_{n m}=a_{n m} e^{2 \pi i v_{n m} t}=a_{n m}^{*} e^{-2 \pi i v_{m n} t}=x_{m n}^{*},
$$

pois $\mathrm{V}_{n m}=\mathrm{T}_{n}-\mathrm{T}_{m}=-\mathrm{V}_{m n}$.

Observemos que $v_{n n}=0$. No quadro dos $x_{n m}$, os termos simétricos em relação à diagonal principal são conjugados; os termos da diagonal principal são constantes e reais.

Heisenberg faz também corresponder ao momento $p$ conjugado de $x$, em substituição à serie de Fourier $p=\sum_{\tau} b_{\tau} \exp (2 \pi i \tau v t)$, um quadro do tipo acima considerado.

A fim de traduzir, na nova mecânica, as relações entre as coordenadas $x$ e $p$ na teoria clássica, torna-se necessário definir as operações elementares a efetuar com os quadros $\left(x_{n m}\right)$. Heisenberg, ainda aí, valeu-se da analogia com as séries de Fourier. 
A soma e o produto de duas séries de Fourier $x_{1}$ e $x_{2}$, contendo as mesmas freqüências, são séries de Fourier cujos coeficientes são dados por

$$
a_{\tau}=a_{\tau}^{(1)}+a_{\tau}^{(2)} ; A_{\tau}=\sum_{\sigma} a_{\sigma}^{(1)} a_{\sigma-\tau}^{(2)} .
$$

Vemos que a soma e a multiplicação das duas séries de Fourier não introduzem nenhuma nova freqüência.

Para os quadros $\left(x_{n m}^{(1)}\right)$ e $\left(x_{n m}^{(2)}\right)$, contendo as mesmas freqüências definem-se a soma $\left(x_{n m}\right)$ e a multiplicação $\left(X_{n m}\right)$ com o auxílio das fórmulas

$$
\begin{aligned}
& x_{n m}=x_{n m}^{(1)}+x_{n m}^{(2)} \\
& X_{n m}=\sum_{k} x_{n k}^{(1)} x_{k m}^{(2)}=\sum_{k} a_{n k}^{(1)} a_{k m \tau}^{(2)} e^{2 \pi i\left(v_{n k}-v_{k m}\right) t} ;
\end{aligned}
$$

nenhuma nova frequiência é introduzida, pois pelo princípio de combinação de Ritz entre as freqüências possíveis, há a relação $\mathrm{V}_{n k}+\mathrm{V}_{k m}=\mathrm{v}_{n m}$.

Estas regras de cálculo vêm mostrar que os quadros $\left(X_{n m}\right)$ podem ser considerados como matrizes cuja teoria, há muito, é conhecida. As matrizes acima indicadas são do tipo de Hermite: $x_{n m}=x_{m n}^{*}$.

A multiplicação das matrizes é distributiva e associativa, mas, em geral, não é comutativa.

Diz-se que uma matriz é diagonal quando todos os seus termos, exceto os da diagonal principal, são nulos:

$$
\left(x_{n m} \delta_{n m}\right)=\left(\begin{array}{cccc}
x_{00} & 0 & 0 & \ldots \\
0 & x_{11} & 0 & \ldots \\
0 & 0 & x_{22} & \ldots \\
\vdots & \vdots & \vdots & \ddots
\end{array}\right),
$$

em que $\delta_{n m}=1$ se $n=m$ e 0 se $n \neq m$.

A matriz

$$
\delta=\left(\delta_{n m}\right)=\left(\begin{array}{cccc}
1 & 0 & 0 & \ldots \\
0 & 1 & 0 & \ldots \\
0 & 0 & 1 & \ldots \\
\vdots & \vdots & \vdots & \ddots
\end{array}\right)
$$

é a matriz unidade. É fácil ver que é uma matriz comutável com uma matriz qualquer $x=\left(x_{n m}\right)$ :

$$
x \delta-\delta x=0 .
$$

A matriz recíproca de $x, x^{-1}$, é definida por $x^{-1} x=\delta ; x^{-1} \mathrm{e}$ $x$ são comutáveis; tem-se $\delta^{-1}=\delta$.

A divisão da matriz $x$ pela matriz $y$ é, por definição, o produto $x y^{-1}$. A matriz $\dot{x}$, derivada em relação ao tempo $t$ da matriz $x$, é a matriz $\dot{x}_{n m}=2 \pi i v_{n m} x_{n m}$.

Se $x$ é diagonal, $\dot{x}=0$. A recíproca desta proposição é verdadeira quando $\mathrm{v}_{n m} \neq 0$, para $n \neq m$, pois neste caso $\dot{x}$ e $x_{n m}$ se anulam ao mesmo tempo; isto acontece, como sabemos, quando o sistema não é "degenerado".

Consideremos a matriz $y$ função ${ }^{2}$ da matriz $x, y=f(x)$. A matriz derivada de $y$ em relação a $x$, é por definição,

$$
\frac{d y}{d x}=\lim _{c=0} \frac{f(x+c)-f(x)}{c}
$$

$c$ designando a matriz diagonal com termos iguais

$$
\begin{aligned}
& c=\alpha \cdot \delta=\left(\begin{array}{cccc}
\alpha & 0 & 0 & \ldots \\
0 & \alpha & 0 & \ldots \\
0 & 0 & \alpha & \ldots \\
\vdots & \vdots & \vdots & \ddots
\end{array}\right) \\
& \operatorname{Se} y=x, \frac{d y}{d x}=\delta \text {. Se } y=\phi(x) \Psi(x), \frac{d y}{d x}=\phi \frac{d \psi}{d x}+\psi \frac{d \phi}{d x} .
\end{aligned}
$$

Podemos agora introduzir algumas noções sobre a Mecânica das Matrizes.

De acordo com as idéias de Heisenberg substituem-se a variável $x$ e o seu momento conjugado $p$ pelas matrizes $\left(x_{n m}\right)$ e $\left(p_{n m}\right)$ cujos elementos dependem das intensidades e das frequiências das radiações emitidas pelo sistema atômico.

Na teoria de Bohr, como vimos, $x$ e $p$ satisfazem às equações canônicas, e a variável de "ação" $J$ verifica a relação $J=\int p \mathrm{~d} x=n h, n$ designando um número inteiro e $h$ a constante de Planck; a integral é tomada ao longo de um ciclo completo de variação de $x$.

Heisenberg substituiu o postulado "mecânico" da teoria de Bohr pela relação

$$
p x-x p=\frac{h}{2 \pi i} \delta
$$

entre as matrizes $x$, $p$, e a matriz unidade $\delta$. Esta relação exprime que as matrizes $x$ e $p$ não são comutáveis e dá a expressão da diferença $p x-x p$.

Vamos mostrar como foi Heisenberg conduzido, pela aplicação do principio de correspondência de Bohr a tal relação que é fundamental na nova Mecânica Quântica.

Temos

$J=\int p d x=\int_{0}^{1 / v} p \dot{x} d t=\int_{0}^{1 / v} d t \sum_{\tau, \sigma} b_{\tau} 2 \pi i v \sigma a_{\sigma} e^{2 \pi i(\tau+\sigma) v t}$,

substituindo $x$ e $p$ pelos seus desenvolvimentos em série de Fourier e atendendo a que $1 / \mathrm{v}$ é o período do movimento.

$\mathrm{Na}$ integração, o termo constante (correspondente a $\tau+\sigma=0$ ) é o único que não fornece um resultado nulo: vem pois

$$
J=-\sum_{\tau} 2 \pi i \tau b_{\tau} a_{-\tau}
$$

$\mathrm{ou}$

$$
1=-2 \pi i \sum_{\tau} \tau \frac{\partial}{\partial J}\left(b_{\tau} a_{-\tau}\right)
$$

Heisenberg, guiado pelo principio de correspondência de Bohr, substituiu sistematicamente quantidades tais como $\mathrm{a}_{\tau}(J), \mathrm{b}_{\tau}(J)$, ou $\mathrm{a}_{\tau}(n h), \mathrm{b}_{\tau}(n h), \mathrm{a}_{n, n-\tau}, \mathrm{b}_{n, n-\tau}$ correspondentes ao salto quântico $n, n-\tau$. Ao operador $h \tau \partial / \partial J$ corresponde, também, na teoria quântica, conforme vimos na últi- 
ma conferência, a diferença $\Delta$ relativa aos estados $n$ e $n-\tau$. Devemos, pois, substituir $h \tau \partial\left(\mathrm{b}_{\tau} \mathrm{a}_{-\tau}\right) / \partial J$ por $\Delta\left(\mathrm{b}_{n, n} \tau \mathrm{a}_{n-\tau, n}\right)$ ou $\mathrm{b}_{n+\tau, n} \mathrm{a}_{n, n+\tau}-\mathrm{b}_{n, n-\tau} \mathrm{a}_{n-\tau, n}$, e obtemos $h / 2 \pi i=\sum_{\tau} \mathrm{b}_{n, n-\tau} \mathrm{a}_{n-\tau, n}-\sum_{\tau} \mathrm{b}_{n+\tau}$ ${ }_{n} \mathrm{a}_{n, n+\tau} ;$ ora a soma deve ser feita tomando os valores inteiros de $\tau$ de $-\infty$ a $+\infty$; a cada número $\tau$ corresponde um outro $-\tau$, de modo que

$$
\frac{h}{2 \pi i}=\sum_{\tau}\left[b_{n, n-\tau} a_{n-\tau, n}-a_{n, n-\tau} b_{n-\tau, n}\right] .
$$

Esta fórmula mostra que o $n$-ésimo termo diagonal da matriz $p x-x p$ é igual a $h / 2 \pi i$, ou ao $n$-ésimo termo da matriz diagonal $(h / 2 \pi i) \delta$.

Heisenberg generalizou este resultado admitindo a coincidência da matriz $p x-x p$ com a matriz $(h / 2 \pi i) \delta$ na Mecânica Quântica.

Observe-se: nos casos em que, nas equações se pode desprezar os termos que contêm $h$ em fator em face dos outros termos, as matrizes $x$ e $p$ se tornam comutáveis e a Mecânica Clássica pode ser utilizada.

O principio de correspondência ainda conduziu Heisenberg a admitir a possibilidade de formar a função hamiltoniana $H(x, p)$ das matrizes $x$ e $p$, tal que $\dot{x}$ e $\dot{p}$ satisfaçam às equações denominadas canônicas

$$
\dot{x}=\frac{\partial H}{\partial p}, \quad \dot{p}=-\frac{\partial H}{\partial x} .
$$

A escolha da matriz $H(x, p)$ faz-se, nos problemas mais simples da Mecânica Quântica, por analogia com a função $H$ da teoria clássica; os resultados confrontados com a experiência têm sido satisfatórios em tais casos.

Observemos que às equações "canônicas" entre as matrizes $x$ e $p$ corresponde uma infinidade de equações do tipo

$$
\begin{aligned}
& 2 \pi i v_{n m} x_{n m}=\left(\frac{\partial H}{\partial P}\right)_{n m}, \\
& 2 \pi i v_{n m} p_{n m}=-\left(\frac{\partial H}{\partial x}\right)_{n m},
\end{aligned}
$$

as quais combinadas com as equações já estabelecidas

$$
\sum_{k}\left[p_{n k} x_{k n}-x_{n k} p_{k n}\right]=\frac{h}{2 \pi i}
$$

conduzem ao cálculo das freqüências $v_{n m}$ e das amplitudes $a_{n m}$ e $b_{n m}$, das vibrações relativas ao sistema atômico.

Das equações entre as matrizes $x$ e $p$ resultam as seguintes proposições:

1) $H$ é uma matriz diagonal, e a equação $\dot{H}=0$ exprime a conservação da energia.

2) Os termos espectroscópicos $T_{n}$ multiplicados por $h$, coincidem, abstração feita de uma constante aditiva, com os termos diagonais $H_{n m}$ da matriz $H$.

Dirac generalizou a teoria de Heisenberg para o caso de um sistema multiperiódico dependendo de $k$ parâmetros $x_{n m}$. Dirac admitiu, neste caso, as seguintes equações da Mecânica dos "Quanta":

a) Equações canônicas: $\dot{x}_{i}=\frac{\partial H}{\partial p_{i}}, \quad \dot{p}_{i}=-\frac{\partial H}{\partial x_{i}}$

b) Condições quânticas (ditas de "permutação"):

$$
\begin{aligned}
& p_{r} x_{r}-x_{r} p_{r}=\frac{h}{2 \pi i} \delta \\
& p_{r} x_{s}-x_{s} p_{r}=0 \\
& p_{r} p_{s}-p_{s} p_{r}=0 \\
& x_{r} x_{s}-x_{s} x_{r}=0 .
\end{aligned}
$$

As Eqs. (20) não são independentes das Eqs. (19). Em rigor, as Eqs. (20) só podem ser consideradas exatas para um instante determinado; as relações de "permutação" nos instantes seguintes devem ser tiradas das Eqs. (19).

\section{Equivalência entre a mecânica ondulatória e a mecânica das matrizes}

A aplicação da Mecânica das Matrizes aos problemas atômicos conduz a cálculos longos, fastidiosos e às vezes, delicados. Observou-se no cálculo dos níveis energéticos, a identidade, para cada problema estudado, de resultados obtidos separadamente pela Mecânica das Matrizes e pela Mecânica Ondulatória. A equivalência matemática entre as duas teorias foi, aliás, demonstrada por Schrödinger e Eckart cujos trabalhos vieram facilitar consideravelmente a resolução dos problemas de Mecânica Atômica.

Pode-se, agora, afirmar que a Mecânica das Matrizes e a Mecânica Ondulatória constituem dois aspectos diferentes da mesma teoria quântica.

Vamos expor, em suas linhas gerais, a teoria de Schrödinger, considerando o caso do campo permanente.

A equação da conservação da energia na Mecânica Clássica se escreve

$$
H\left(x_{i}, p_{i}\right)-E=0 .
$$

Sabemos que $H=T+U$. Em coordenadas curvilíneas temos a equação da conservação da energia sob a forma dita "simetrizada"

$$
\frac{1}{2 m} \frac{1}{\sqrt{a}} \sum_{i} p_{i} \sqrt{a} \sum_{j} a^{i j} p_{j}+U\left(x_{i}\right)-E=0 .
$$

Substituamos $p$ pelo operador $(h / 2 \pi i) \partial / \partial x_{i} ; E$, pelo operador $(h / 2 \pi i) \partial / \partial t$, e $x_{i}$ pelo operador "multiplicação por $x_{i}$ "; apliquemos o operador resultante, assim obtido, à função $\Psi$ das ondas; vem

$$
\left[H\left(x_{i}, \frac{h}{2 \pi i} \frac{\partial}{\partial x_{i}}\right)-\frac{h}{2 \pi i} \frac{\partial}{\partial t}\right] \Psi=0
$$

$\mathrm{ou}$

$$
\nabla^{2} \Psi-\frac{4 \pi m i}{h} \frac{\partial \Psi}{\partial t}-\frac{8 \pi^{2} m}{h^{2}} U \Psi=0
$$

equação de Schrödinger.

Se o campo é permanente, a equação de Schrödinger pode ser escrita 


$$
\left[H\left(x_{i}, \frac{h}{2 \pi i} \frac{\partial}{\partial x_{i}}\right)-E\right] \Psi=0 .
$$

Neste caso, se $E_{n}$ são os valores fundamentais ou característicos, e $\Psi_{n}$ as soluções correspondentes, podemos supor o sistema $\operatorname{dos} \Psi_{n}$ normal e ortogonal

$$
\int \Psi_{n} \Psi_{m}^{*}=\delta_{n m}
$$

Conforme já dissemos em outra conferência, uma função contínua $f(\mathbf{r})$ pode, sob certas condições, ser desenvolvida em série de funções características,

$$
f(\mathbf{r})=\sum_{n} C_{n} \Psi_{n}(\mathbf{r}),
$$

sendo

$$
C_{n}=\int \Psi_{n} f d^{3} r
$$

a integração sendo estendida a todo o espaço.

Schrödinger observou que as condições (20) da Mecânica das Matrizes conduzem a relações entre operadores diferenciais lineares relativamente a $x_{i}$. Assim, por exemplo, no caso de um sistema que depende de um parâmetro, substituindo na equação fundamental $p x-x p=(h / 2 \pi i) \delta, p$ por $(h / 2 \pi i) \delta / \partial x$, $\delta$ pela unidade 1 , e considerando $x$ como operador, vem

$$
\frac{\partial}{\partial x} x-x \frac{\partial}{\partial x}=1
$$

que é verificada identicamente, pois

$$
\frac{\partial}{\partial x}(x \Psi)-x \frac{\partial \Psi}{\partial x}=\Psi .
$$

Schrödinger procurou, então, descobrir uma conexão entre as relações entre operadores, equivalentes às equações das matrizes, e as relações análogas equivalentes às equações das ondas.

Seja $F(x, p)$ uma função das variáveis $x$ e $p$ (Mecânica Clássica). Substituamos em $F, p$ pelo operador $(h / 2 \pi i) \partial / \partial x$ e consideremos $x$ como operador ("multiplicação por $x$ "). $F(x,(h / 2 \pi i) \partial / \partial x)$ torna-se um operador que aplicado a $\Psi_{n}$ dá uma função de $x$ que, desenvolvida em série de funções fundamentais, conduz a

$$
F\left(x, \frac{h}{2 \pi i} \frac{\partial}{\partial x}\right) \Psi_{n}=\sum_{m} F_{n m} \Psi_{m} .
$$

Os coeficientes $F_{n m}$ são dados por

$$
F_{n m}=\int \Psi_{n}^{*} F\left(x, \frac{h}{2 \pi i} \frac{\partial}{\partial x}\right) \Psi_{m} d^{3} r
$$

Para $x$ e $p$, tem-se, respectivamente,

$$
\begin{aligned}
& x_{n m}=\int \Psi_{n}^{*} x \Psi_{m} d^{3} r \\
& p_{n m}=\frac{h}{2 \pi i} \int \Psi_{n}^{*} \frac{\partial \Psi_{m}}{\partial x} d^{3} r .
\end{aligned}
$$

Schrödinger demonstrou: 1) que os quadros formados com os coeficientes tais como $F_{n m}$, multiplicados por $\exp \left(2 \pi i v_{n m} t\right)$ verificam as regras do cálculo das matrizes e podem ser considerados como matrizes de Heisenberg correspondentes às funções $F(x . p) ; 2)$ as matrizes $\left(x_{n m}\right)$ e $\left(p_{n m}\right)$ satisfazem às equações da Mecânica das Matrizes, e tornam portanto a matriz $H$ diagonal.

Descoberta a conexão existente entre a Mecânica Ondulatória e a Mecânica das Matrizes, impõe-se, como sendo geralmente o mais cômodo, o seguinte processo para resolver um problema de Mecânica Atômica:

Forma-se em primeiro lugar a equação da Mecânica Ondulatória, partindo-se da função de Hamilton $H$. Os valores característicos da equação das ondas representam os níveis energéticos $E_{n}$ do sistema. Conhecendo-se as soluções características, as fórmulas há pouco estabelecidas permitem calcular os elementos das matrizes que representam as variáveis $x, p$, etc., e portanto as intensidades e os estados de polarização das radiações emitidas pelo sistema atômico, bem como suas constantes mecânicas.

Vemos que as soluções características $\Psi_{n}$ são meras funções intermediárias no cálculo dos elementos das matrizes $x, p$, etc. elementos esses que representam as únicas grandezas cuja significação experimental é precisa, e cuja obtenção deve constituir o principal objetivo da Mecânica dos "Quanta". 